\title{
Princípios de Hong Kong para a Avaliação de Pesquisadores: Promovendo a Integridade em Pesquisa
}

\author{
David Moher, Lex Bouter, Sabine Kleinert, Paul Glasziou, Mai Har Sham, Virginia Barbour, \\ Anne-Marie Coriat, Nicole Foeger, Ulrich Dirnagl \\ Publicado em 16 de julho de 2020, https://doi.org/10.1371/journal.pbio.3000737
}

\author{
Tradução e adaptação para a língua portuguesa: Sonia Vasconcelos (IBqM/UFRJ), \\ Carmen Penido (FIOCRUZ), Sigmar de Mello Rode (UNESP/ABEC Brasil) \\ Publicado em janeiro de 2021, https://doi.org/10.21452/abec.2021.abec.001
}

\begin{abstract}
Resumo: Para que o conhecimento beneficie a pesquisa e a sociedade, ele deve ser confiável. A pesquisa confiável é robusta, rigorosa e transparente em todas as etapas relativas ao desenho, execução e relato. Entretanto, a inclusão de considerações relacionadas à confiabilidade, ao rigor e à transparência na avaliação de pesquisadores continua sendo rara. Os Princípios de Hong Kong (HKPs) foram desenvolvidos como parte da $6^{\text {a }}$ Conferência Mundial sobre Integridade em Pesquisa, com foco sobre a necessidade de impulsionar o aprimoramento da pesquisa, garantindo que os pesquisadores sejam explicitamente reconhecidos e recompensados por atitudes que fortalecem a integridade. Apresentamos cinco princípios: práticas de pesquisa responsáveis; relato transparente; ciência aberta (pesquisa aberta); valorização da diversidade de tipos de pesquisa; reconhecimento de todas as contribuições para a pesquisa e atividade acadêmica. Para cada princípio, oferecemos uma justificativa, com exemplos que ilustram onde esses princípios já estão sendo adotados.
\end{abstract}

Citação: Moher D, Bouter L, Kleinert S, Glasziou P, Sham MH, Barbour V, et al. (2020) The Hong Kong Principles for assessing researchers: Fostering research integrity. PLoS Biol 18(7): e3000737. https://doi.org/10.1371/journal.pbio.3000737

Copyright: ( 2020 Moher et al. Este é um artigo de acesso aberto distribuído sob os termos da Licença de Atribuição Creative Commons Attribution License, que permite o uso, distribuição e reprodução irrestritos em qualquer meio, na condição de que o autor original e a fonte sejam creditados.

Financiamento: O autor PG é financiado pelo Conselho Nacional de Saúde e Pesquisa Médica (National Health and Medical Research Council) (NHMRC) da Austrália, Auxílio APP1155009. Os financiadores não tiveram nenhum papel no desenho do estudo, na coleta e análise de dados, na decisão de publicar ou na preparação do manuscrito.

Interesses Concorrentes: Eu li as políticas do periódico e declaro que os autores deste manuscrito têm os seguintes interesses concorrentes: A-MC trabalha para a Wellcome. Por meio desse vínculo, a organização e a autora estão envolvidas em várias ações para defender e promover uma cultura de pesquisa mais saudável. As orientações do periódico fazem referência a esse tipo de atividade e, por essa razão, a inclusão foi feita. VB esteve envolvida na criação de um curso de integridade em pesquisa na QUT [Queensland University of Technology], que disponibiliza a licença para outras instituições. Com essa disponibilização, uma parte da receita é destinada aos responsáveis pela criação do curso. VB tem vínculo empregatício com a QUT e com o Australasian Open Access Strategy Group; a mesma autora é membro do Comitê Gestor sobre Qualidade de Pesquisa do NHMRC, sendo essa uma atividade remunerada. VB é consultora, sem vínculo financeiro, para várias iniciativas sobre acesso aberto e comunicação acadêmica, incluindo a DORA.

Abreviaturas: DMP, data management plan; DOI, digital object identifier; DORA, Declaration on Research Assessment; DR-NTU, NTU Digital Repository; EDA, Experimental Design Assistant; FAIR, Findable, Accessible, Interoperable, and Re-usable; HKPs, Hong Kong Principles; HRB, Health Research Board; NC3Rs, National Centre for the Replacement, Refinement \& Reduction of Animals in Research; NIH, National Institutes of Health; NIHR, National Institute of Health Research; NTU, Nanyang Technological University; ORCID, Open Researcher and Contributor ID; PTC, promotion and tenure committee; REWARD, REduce research Waste And Review Diligence; SPOR, Strategy for Patient-Oriented Research; WCRI, World Conference on Research Integrity; WOR, Wellcome Open Research

Tipo de Contribuição: Não comissionada; revisada externamente por pares [peer review]. 


\section{INTRODUÇÃO}

Em uma busca pelo avanço do conhecimento, os pesquisadores publicam, aproximadamente, 1,5 milhão de artigos por ano em periódicos. O pressuposto é que essa literatura pode ser usada por outros pesquisadores, outros atores e pela sociedade em geral, por ser uma literatura confiável, robusta, rigorosa e completa.

A abordagem adotada para validar a pesquisa, bem como seus resultados, varia, dependendo da sua natureza. Por exemplo, para examinar rigorosamente os efeitos de uma intervenção em saúde, os participantes do estudo (humanos ou animais), normalmente, precisam ser randomizados. Muitos pesquisadores defendem que haja um registro de protocolos como uma estratégia para garantir a transparência e reduzir vieses, permitindo que seja possível discernir entre modos exploratórios e confirmatórios de pesquisa. Esse registro também oferece uma visão sobre os projetos de pesquisa em andamento. Posteriormente, o uso de diretrizes para a elaboração do relato científico pode ser um auxílio para assegurar que os pesquisadores apresentem um relato completo e transparente dos métodos e dos resultados. Quando a pesquisa está sendo divulgada, a equipe deve garantir que os dados, os materiais e qualquer código de análise sejam disponibilizados como parte integrante da publicação. O compartilhamento de dados facilita a reanálise do material para verificar a reprodutibilidade e para realizar análises secundárias.

Embora haja alguns mecanismos que auxiliam os pesquisadores a garantir a transparência em todos os estágios da pesquisa, seu desenho, execução e relato, não há uma adoção generalizada dessas práticas em todas as áreas do conhecimento. Há várias razões interligadas nessa questão. Argumentamos que um fator a ser considerado é a pouca ênfase destinada ao rigor científico, ao contratar, avaliar e promover pesquisadores. Nossa impressão é que a ênfase é maior na novidade do "impacto" percebido da pesquisa do que no rigor científico [2]. Acreditamos que o trabalho conjunto para tratar dessa questão sistêmica, nos mais variados contextos de pesquisa, ofereceria uma oportunidade, no cenário global, para aprimorar a atividade científica e seu impacto.

Dessa forma, desenvolvemos os Princípios de Hong Kong (HKPs), como parte da $6^{\text {a }}$ Conferência Mundial sobre Integridade em Pesquisa (WCRI). O intuito é gerar maior reconhecimento para pesquisadores que se comprometem com práticas robustas, rigorosas e transparentes (i.e., suas carreiras avançariam com essas práticas) (Fig. 1). Se implementados, os HKPs podem desempenhar um papel crucial nas avaliações de pesquisadores, baseadas em evidências, e colocar o rigor científico no centro da avaliação, além de abrir os dados de pesquisa para a sociedade, que se beneficiaria mais amplamente. 
Figura 1. Indicadores de práticas de pesquisa responsáveis

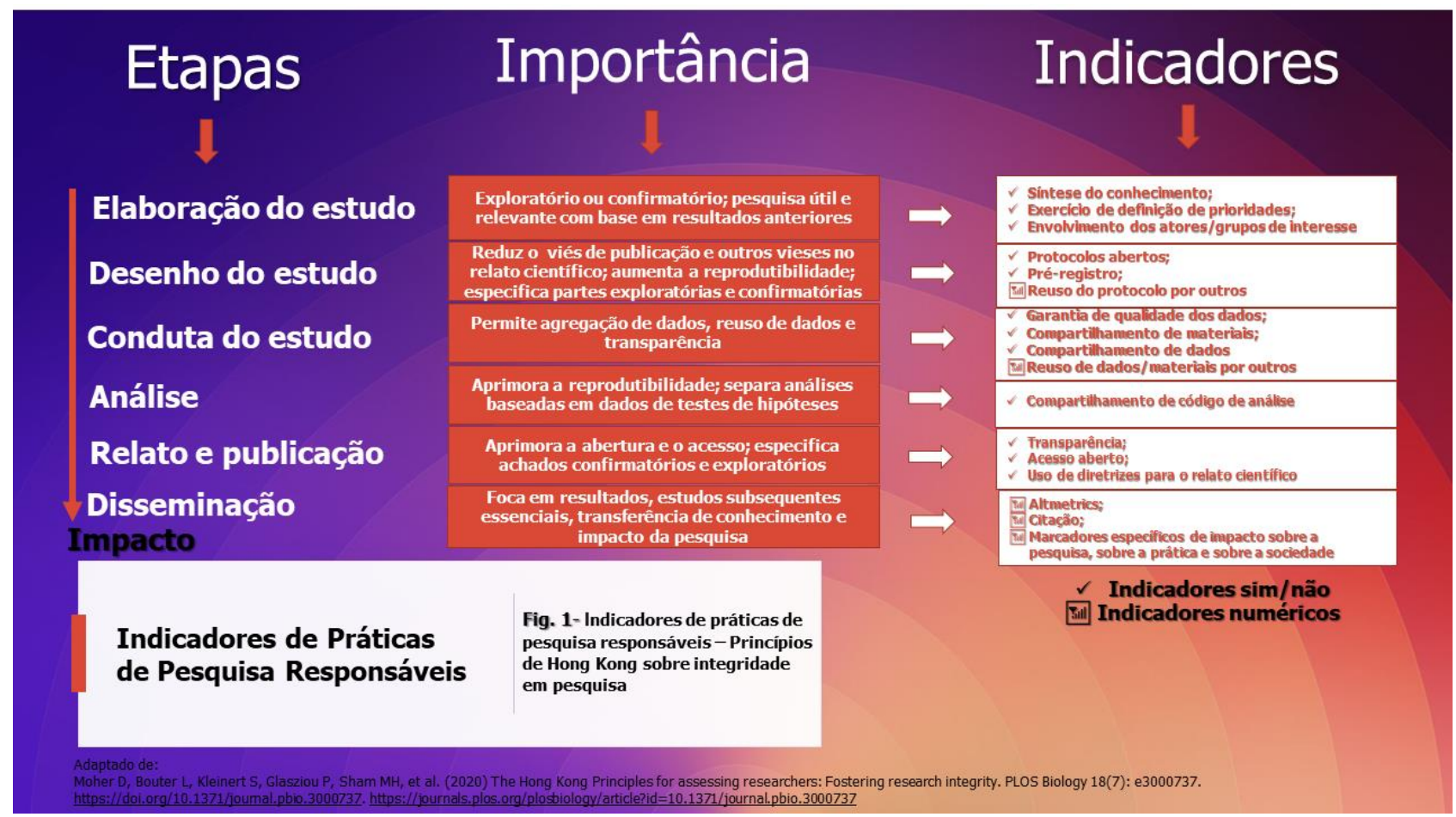

Propomos cinco princípios, cada um com uma justificativa associada à sua inclusão. Os princípios contemplam pesquisas e análises de natureza exploratória e confirmatória. Da mesma forma, esses princípios são aplicáveis à pesquisa quantitativa e qualitativa, embora haja mais foco na avaliação de pesquisadores que se dedicam à pesquisa empírica. Os princípios foram formulados enfatizando recompensas/reconhecimento por atitudes que fortalecem a integridade, assim como privilegiando práticas de pesquisa responsáveis e a prevenção de práticas danosas à ciência [3]. Ilustramos esses princípios com exemplos que são do nosso conhecimento. Esses exemplos não são exaustivos e muitos são relevantes para mais de um princípio. No conjunto, ilustram uma variedade de abordagens de como os princípios podem impactar os níveis mais elevados da pesquisa internacional.

As primeiras versões dos HKPs foram compartilhadas com os 700 participantes registrados na $6^{\mathrm{a}}$ WCRI. Outras discussões aconteceram durante duas sessões na $6^{\mathrm{a}}$ WCRI. Uma penúltima versão foi postada no website da $6^{\mathrm{a}}$ WCRI após a conferência. Mais de 100 pessoas contribuíram com informações e feedback. Reconhecemos todas essas contribuições valiosas e a liderança global daqueles que trabalharam na Declaração de São Francisco sobre Avaliação da Pesquisa (DORA), no Manifesto de Leiden e em outras iniciativas para promover o uso responsável de métricas, que constituem a fundação de muito do que se baseia o nosso trabalho [2,4,5,6,7]. Os HKPs são formulados a partir da perspectiva da comunidade de integridade em pesquisa.

Nós, assim como os signatários da DORA, acreditamos fortemente que as métricas atuais podem atuar como incentivos perversos na avaliação de pesquisadores. No entanto, o enfoque dos princípios aqui delineados é sobre os prejuízos que esse contexto traz à integridade em pesquisa [8]. A seguir, usamos versões abreviadas do texto dos HKPs para facilitar a disseminação dos princípios. A redação completa de cada princípio está apresentada no Quadro 1. 
Quadro 1. Redação completa dos HKPs

Princípio 1: Deve-se valorizar, na avaliação dos pesquisadores, as práticas responsáveis desde a concepção até a execução da pesquisa, incluindo o desenvolvimento da ideia inicial do trabalho, o desenho de pesquisa, metodologia, execução e a disseminação efetiva dos resultados.

Princípio 2: Deve-se valorizar o relato preciso e transparente das pesquisas, independentemente dos seus resultados.

Princípio 3: Deve-se valorizar as práticas da ciência aberta (pesquisa aberta) - como métodos, materiais e dados abertos.

Princípio 4: Deve-se valorizar um amplo espectro de pesquisas e contribuições acadêmicas, como replicação, inovação, translação, síntese e metapesquisa.

Princípio 5: Deve-se valorizar uma série de outras contribuições para fomentar a pesquisa responsável e para a atividade acadêmica, como a revisão por pares para projetos e publicações, a orientação, a divulgação/extensão e a troca de conhecimento.

\section{PRINCÍPIOS}

\section{Princípio 1: Avalie as práticas de pesquisa responsáveis}

\section{Justificativa}

Com frequência, o número de publicações, citações e o volume total de projetos financiados ainda são as métricas dominantes usadas por instituições de pesquisa para avaliar e recompensar seus pesquisadores $[2,4,5,6]$. Oferecer bônus a acadêmicos pela publicação em determinados periódicos (i.e., pagamento por mérito) também é comum em muitas partes do mundo [9-11]. Esses critérios de avaliação dizem pouco aos avaliadores sobre os pesquisadores e sobre o rigor das suas pesquisas. Não seriam, portanto, métricas necessariamente "responsáveis", embora pesquisas citadas milhares de vezes provavelmente indiquem que essas citações seriam alguma medida de impacto. Essas métricas também podem ser indevidamente influenciadas por áreas de pesquisa e práticas de citação, fornecendo poucas informações sobre as contribuições de uma publicação (e, portanto, de um pesquisador) para a pesquisa e a sociedade. Outros critérios são necessários para proporcionar uma visão mais ampla sobre os indicativos de boas práticas: um exemplo é até que ponto um pesquisador envolve determinados membros do público no desenvolvimento de perguntas de pesquisa (Fig. 1). Os pesquisadores envolvidos com práticas de pesquisa responsáveis, como o compartilhamento de dados, que inclusive pode consumir mais tempo e recursos, podem ficar em desvantagem, se comparados a colegas que não adotam essas práticas. As avaliações de carreira precisam reconhecer esse problema.

\section{Implementações em curso}

A Estratégia para a Pesquisa Orientada para o Paciente dos Institutos Canadenses de Pesquisa em Saúde (Canadian Institutes of Health Research's Strategy for Patient-Oriented Research) (SPOR) é uma iniciativa multimilionária. A SPOR proporciona aos pacientes uma ampla gama de atividades relacionadas à pesquisa nas províncias e territórios canadenses [12]. Os pacientes se envolvem ativamente no desenvolvimento de projetos de pesquisa, estabelecendo prioridades e formulando perguntas de pesquisa. A resposta de Ontário (Ontario SUPPORT Unit) incluiu uma série de artigos com esses atores, que assumem um papel de liderança na coautoria do conteúdo do trabalho [13]. No Reino Unido, a James Lind Alliance, financiada pelo Instituto Nacional de Pesquisa em Saúde (National Institute of Health Research) (NIHR) do Reino Unido, é um exemplo de sucesso na inclusão de pacientes, cuidadores e médicos no desenvolvimento de parcerias para a definição de 
prioridades [14] e formulação de perguntas [15]. Há outros exemplos de ciência cidadã em diferentes áreas do conhecimento [16].

Com foco sobre o aumento da reprodutibilidade, os Institutos Nacionais de Saúde (National Institutes of Health) (NIH) dos Estados Unidos revisaram suas instruções para submissão e critérios de revisão de projetos para fortalecer o rigor científico e a transparência [17]. Um dos recursos que o NIH recomenda é o Assistente de Desenho Experimental (Experimental Design Assistant) (EDA), desenvolvido pelo Centro Nacional para a Substituição, Refinamento e Redução de Animais em Pesquisa (National Centre for the Replacement, Refinement and Reduction of Animals in Research) (NC3Rs). Essa ferramenta online de 10 módulos tem o intuito de auxiliar os pesquisadores no projeto e análise de experimentos com animais. A mesma ferramenta inclui suporte dedicado para a randomização, cegamento e cálculo amostral. Além disso, a ferramenta pode ser usada para ajudar os pesquisadores a preparar as informações do projeto experimental e o plano de análise solicitado para os pedidos de financiamento [18]. A EDA é uma das várias ferramentas disponíveis que auxiliam os pesquisadores nos esforços para garantir o rigor das propostas de pesquisa e da própria condução do projeto, de forma geral.

Outros exemplos de critérios alternativos incluem: métricas de mídia social como indicadores de divulgação científica [19], apresentações públicas sobre resultados, engajamento público, bem como outros tipos de eventos envolvendo financiadores, pesquisadores e outros atores para um plano de comunicação eficaz associado ao programa de pesquisa [20]. Organizações como Wellcome Trust estão tendo uma atitude holística para redefinir abordagens de engajamento explicitamente para auxiliar o público a desenvolver um sentimento de empoderamento para acessar, usar, responder e criar pesquisas em saúde [21].

\section{Princípio 2: Valorize o relato completo}

\section{Justificativa}

A publicação incompleta do conjunto de resultados distorce seriamente a base de evidências para a tomada de decisão. Uma revisão sistemática de estudos com reboxetina para o tratamento da depressão ilustra essa questão. Essa revisão identificou que quase três quartos dos pacientes incluídos estavam em estudos que não tinham sido publicados [22]; há outros exemplos em diferentes áreas do conhecimento [23,24]. A publicação seletiva de pesquisas com resultados positivos (i.e., viés de publicação) distorce a base de evidências científicas e foi demonstrada em diversas áreas, incluindo economia, psicologia, pesquisa clínica e pré-clínica em saúde (e.g., [25]). Além disso, a frequência de outros vieses nos relatos científicos (e.g., resultados primários alterados sem divulgação, bem como spin) é de cerca de 30\% [26]. Esse percentual é inaceitavelmente alto e diminui a confiabilidade e a integridade da pesquisa [11]. Aparentemente, até os Comitês de Promoção e Progressão na Carreira (Promotion and Tenure Committees) (PTCs) geralmente não dão importância suficiente ao registro de protocolos e planos de análise de dados, à publicação completa de estudos concluídos ou à disponibilização de dados, códigos e materiais [27]. Essas atividades merecem crédito na avaliação de pesquisadores porque são essenciais à replicabilidade, o que possibilita verificar o que foi feito, bem como viabilizar o reaproveitamento dos dados. 


\section{Implementações em curso}

Diretrizes para o registro de estudos e para a elaboração do relato científico são ferramentas úteis para ajudar a melhorar a integridade e a transparência de um amplo espectro de pesquisas [28-31]. Como parte das políticas editoriais da plataforma de publicação de acesso aberto da Wellcome Trust (Wellcome Open Research [WOR]), os autores são obrigados a usar diretrizes para a elaboração do relato científico ao enviar protocolos para um estudo (e.g., SPIRIT) e estudos concluídos (e.g., ARRIVE) [32]. Outros financiadores, como o Gates Open Research [33], o NC3Rs Gateway [34] e a Association of Medical Research Charities [35], fazem o mesmo. Para ajudar a reduzir o viés de publicação, a WOR e outros periódicos [36,37] usam relatórios registrados [38] (consulte a aba Participating journals). Da mesma forma, para promover o registro e a publicação de todas as pesquisas, o NIHR no Reino Unido indica que "ao submeter aos programas NIHR para financiamento de um novo estudo clínico, o proponente deve divulgar/disponibilizar publicações anteriores e histórico de registros de ensaios clínicos relacionados a quaisquer publicações relevantes, assim como financiamentos de pesquisa obtidos, referenciados na submissão" [39]. Embora esses sejam exemplos de boas práticas de financiadores, poucas instituições de pesquisa as incorporaram nas avaliações de pesquisadores $[27,40,41]$.

Diversas instituições de pesquisa (e.g., Universidade de Toronto) estão agora recomendando que seus pesquisadores clínicos usem o SEPTRE [42], uma ferramenta de gerenciamento e criação de protocolo baseada na web. Quando o SEPTRE é usado, as informações do protocolo para os ensaios são automaticamente registradas no clinicaltrials.gov. Essa opção economiza tempo e auxilia os pesquisadores e suas instituições de pesquisa a manter as boas práticas de publicação (e.g., registro de ensaios). Alguns periódicos em ciências sociais, particularmente na área de psicologia, usam relatórios registrados para ajudar a garantir que a pesquisa seja publicada, independentemente de seus resultados $[43,44]$.

\section{Princípio 3: Recompense a prática da ciência (pesquisa) aberta}

\section{Justificativa}

A transparência na pesquisa (e.g., acesso aberto, métodos abertos, dados abertos, códigos abertos) representa mais do que o simples acesso à pesquisa - ela traz igualdade ao processo científico. A ciência aberta compreende um conjunto de práticas em todo o ciclo da pesquisa [45]. Quando falamos sobre o acesso à pesquisa, o foco da discussão não deve ser sobre quem pode custear o acesso às publicações, como é o caso dos periódicos científicos com assinaturas pagas. Decisões sobre assistência à saúde e políticas sociais devem ser fundamentadas no acesso integral, e não parcial, ao conhecimento científico [46]. Uma parcela considerável dos recursos públicos é destinada à pesquisa, e seus resultados devem ter grande impacto social. Pesquisadores da área pré-clínica estão se comprometendo a compartilhar abertamente seus cadernos de laboratório [47] para agilizar a pesquisa, otimizar colaborações e reduzir duplicações desnecessárias.

Com o intuito de desencorajar práticas questionáveis de autoria, o Consortia Advancing Standards in Research Administration Information apoia o uso do sistema de taxonomia CRediT [48], como meio de os autores descreverem de forma mais transparente a sua contribuição para o projeto de pesquisa. O compartilhamento de dados é outro exemplo de práticas abertas, mas não é uma prática comum na 
pesquisa clínica (com algumas exceções, como na genética) [49], embora os participantes da pesquisa pareçam apoiar o compartilhamento de seus dados, pelo menos os dos ensaios randomizados dos quais participaram [50]. O compartilhamento de dados também não é uma prática estabelecida em muitas outras áreas do conhecimento. Sem o compartilhamento de dados, é difícil verificar a seletividade de dados e informações nos relatos de pesquisa; o compartilhamento de dados é fundamental para abordar as questões referentes à reprodutibilidade [51] e à construção de confiança [1]. Existem diferentes estimativas sobre o quanto da pesquisa é disponibilizado por veículos de acesso aberto, tais como periódicos, repositórios ou preprints, mas está longe de ser 100\% [52]. Parece claro que as diferentes modalidades de ciência aberta precisam ser recompensadas na avaliação de pesquisadores, pois essas práticas contribuem fortemente para aumentar a transparência, que é um princípio fundamental da integridade em pesquisa $[45,53]$.

\section{Implementações em curso}

A Universidade de Ghent, na Bélgica, adotou diretrizes para o compartilhamento de dados: "O gerenciamento de dados sólidos é um requisito básico para esta [análise acadêmica] e oferece garantias adicionais para metodologias confiáveis, compartilhamento e reutilização de dados por outros pesquisadores em um contexto de Ciência Aberta, assim como para a responsabilidade da integridade acadêmica do próprio pesquisador" [54]. A Universidade Tecnológica de Nanyang (NTU), Singapura, implementou uma Política de Acesso Aberto em 2011. Todo o corpo docente e os funcionários da NTU devem depositar a versão final dos manuscritos de seus artigos revisados pelos pares e aqueles apresentados em conferências no Repositório Digital (DR-NTU) mantido pela biblioteca, após a aceitação de suas publicações. Na faculdade de medicina da NTU, são realizadas auditorias aleatórias nos planos de gerenciamento de dados (data management plans) (DMPs) submetidos (obrigatório) e também são feitas verificações para conferir se os dados finais estão realmente compartilhados no repositório de dados de acesso aberto DR-NTU da NTU. Uma coalisão de financiadores foi estabelecida para implementar a publicação de acesso aberto para um futuro próximo [55].

Para ajudar a facilitar o compartilhamento de dados, a Universidade de Cambridge introduziu o conceito de "campeões de dados" [56], em que voluntários aconselham os membros da comunidade de pesquisa sobre o manuseio adequado de dados de pesquisa, incentivando o uso dos princípios de pesquisa FAIR (Findable, Accessible, Interoperable, and Re-usable), i.e., Rastreável, Acessível, Interoperável e Reutilizável [57]. A Universidade de Tecnologia de Delft, na Holanda, levou esse conceito adiante e o implementou como um critério de avaliação de carreira [58]. Os critérios de promoção acadêmica da Universidade de Glasgow explicitamente consideram como produtividade científica o compartilhamento de dados (para promover a replicação) [59].

Alguns periódicos também estabeleceram políticas sólidas de compartilhamento de dados. Por exemplo, os periódicos da PLOS "exigem que os autores tornem totalmente disponíveis todos os dados nos quais se basearam as conclusões descritas em seus manuscritos, sem restrição, no momento da publicação. Quando houver requisitos legais ou éticos específicos que proíbam o compartilhamento público de um conjunto de dados, os autores devem indicar como os pesquisadores podem ter acesso aos dados. A recusa em compartilhar dados e metadados, assim como métodos, de acordo com esta política, será motivo para rejeição" [60]. A iniciativa Promoção da Abertura e 
Transparência do Center for Open Science (The Center for Open Science's Transparency and Openness Promotion) fornece informações sobre os padrões de transparência de dados para uma grande variedade de periódicos [61]. Considerando que o benefício social é parte de uma avaliação de carreira emergente, os pesquisadores da área clínica também devem responder à percepção crescente de que os pacientes desejam que seus dados sejam compartilhados [50].

A pesquisa aberta é promovida pela conformidade com infraestruturas básicas, tais como a exigência de um identificador digital ORCID (Open Researcher and Contributor Identifier) para que cada pesquisador possa ser individualmente identificado. Uma carta recente de financiadores internacionais comprometendo-se a implementar ORCIDs para todos os pesquisadores é um avanço significativo [62]. Isso foi implementado recentemente no Instituto de Pesquisa do Hospital de Ottawa. Na Austrália e na Nova Zelândia, existe um consórcio que adota o ORCID nacionalmente.

O NIH considera preprints em pedidos de financiamento [63], assim como todos os principais financiadores públicos do Reino Unido (e.g., Medical Research Council, UK) [64]. A Wellcome Trust tornou os preprints obrigatórios para o trabalho com foco em emergências em saúde e encoraja amplamente o seu uso, especialmente para pesquisadores em início de carreira [65].

\section{Princípio 4: Reconheça uma variedade de atividades de pesquisa}

\section{Justificativa}

Um sistema que recompensa benefícios à sociedade e estimula pesquisas confiáveis e relevantes deve considerar os diferentes tipos de atividade de pesquisa: criar novas ideias; testá-las; replicar as principais descobertas; resumir pesquisas existentes; desenvolver e validar novas ferramentas, medidas ou métodos etc. Diferentes indicadores e critérios que sejam relevantes para esses diferentes tipos e estágios de pesquisa precisam ser desenvolvidos (Fig. 1). Isso inclui diferentes cronogramas de avaliação para diferentes tipos de pesquisa.

Os incentivos que alimentam uma ideia fixa de que há um "tipo certo" de pesquisa retardam, ou até impedem, o seu progresso. Pesquisas que se baseiam em descobertas acidentais ou pesquisas orientadas pela curiosidade, com base no pensamento "fora da caixa", também devem ser possíveis e encorajadas em um sistema acadêmico de recompensas que valoriza o progresso societário [66]. Por exemplo, a descoberta do grafeno na Universidade de Manchester, Reino Unido, foi resultado de discussões às tardes de sexta-feira, fora das atividades "normais" de pesquisa [67]. Agências financiadoras também estão encorajando submissões multidisciplinares de alto risco [68]. O curto prazo dos ciclos de recompensa na academia torna esse tipo de pesquisa menos atraente para financiadores, instituições e pesquisadores.

Da mesma forma, estudos de replicablidade científica ou de revisão da literatura, muitas vezes, não são considerados inovadores o suficiente nas avaliações da produtividade de pesquisadores. Esse fato é observado, apesar da grande importância dessas contribuições para a credibilidade da pesquisa ou para uma apresentação sistemática equilibrada e robusta das evidências disponíveis [51,69]. Essa questão não é universalmente apreciada pelos Comitês de Promoção e Progressão na Carreira, os PTCs. 
A pesquisa sobre a pesquisa, a metapesquisa, é praticada, por exemplo, por organizações como METRICS (Stanford, CA, EUA) [70], QUEST (Berlim, Alemanha) [71] (com enfoque na metapesquisa clínica e pré-clínica), no Centro de MetaPesquisa da Universidade de Tilburg [72] (Tilburg, Holanda) (com enfoque nas ciências sociais e na Colaboração para a Ciência Aberta - Open Science Collaboration), tal como o Acelerador de Ciências da Psicologia (Psychology Science Accelerator). Essa iniciativa em andamento consiste em colaboradores de centenas de universidades e organizações independentes, sem fins lucrativos, que trabalham para avaliar as barreiras à replicabilidade em psicologia, na biologia do câncer pré-clínico e nas várias áreas das ciências sociais [73]. Essas atividades são importantes para informar e aprimorar as práticas de pesquisa e, portanto, contribuem para torná-la mais confiável e relevante. A questão é que sabemos muito pouco sobre os fatores que motivam práticas de pesquisa responsáveis e as que são danosas à ciência. Além disso, a pesquisa sobre a pesquisa (também conhecida como metapesquisa) ainda é subfinanciada. Assim sendo, é importante que esse tipo de contribuição científica tenha reconhecimento explícito na avaliação de pesquisadores.

\section{Implementações em curso}

Alguns financiadores já reconheceram a relevância de uma ampla gama de atividades científicas. A Research Impact Assessment Platform (Researchfish) trabalha captando parte dessa diversidade e pode gerar relatórios sobre o impacto de um amplo espectro de pesquisas financiadas [74]. $\mathrm{O}$ Wellcome Success Framework destaca a importância de uma visão de longo prazo e de objetivos compartilhados, a fim de adotar uma abordagem mais equilibrada para a avaliação [75]. O Ministério Federal da Ciência e Educação da Alemanha está financiando estudos pré-clínicos confirmatórios [76].

A Wellcome Trust desenvolveu uma nova Estratégia de Estudos Longitudinais Populacionais, financiando prêmios para a reutilização de dados [77] e apoiando a pesquisa sobre a pesquisa [78]. Todas as abordagens têm como objetivo avaliar uma ampla gama de estudos e maximizar o valor da atividade de pesquisa. A Organização Holandesa de Pesquisa Científica (The Netherlands Organization for Scientific Research) está em sua terceira chamada para estudos de replicação [79].

A pesquisa sobre a pesquisa, a metapesquisa, também vem ganhando força e agora com alguns espaços formais no sistema de publicações. Por exemplo, PLOS Biology e eLIFE têm uma seção de metapesquisa [80,81]. Não encontramos nenhuma instituição acadêmica que tenha incorporado replicação ou metapesquisa em seu portfólio de avaliação de carreira [27]. Os NIHR solicitam uma revisão sistemática sobre o problema a ser investigado antes que uma nova pesquisa seja financiada [82]. Os NC3Rs também vêm estimulando a importância das revisões sistemáticas para justificar as propostas de projetos [83,84]. Caso não existam tais revisões, a organização concede financiamento para realizá-las.

\section{Princípio 5: Reconheça outras tarefas essenciais, como revisão por pares e orientação}

\section{Justificativa}

Conforme discutido no Princípio 1, as avaliações de pesquisa frequentemente se concentram em um espectro limitado de métricas que são fáceis de medir, incluindo publicações, citações e financiamento [2,27]. Para que o ecossistema de pesquisa funcione de maneira eficiente, outras 
atividades científicas também são essenciais. A revisão por pares continua sendo a base fundamental da qualidade da avaliação de financiamentos, publicações e conferências. A qualidade das revisões por pares feitas para periódicos e financiadores também deve fazer parte das avaliações de promoção na carreira e de produtividade. Também devem compor esse leque diversas contribuições para a infraestrutura, supervisão ou regulação da pesquisa. Da mesma forma, contribuições que vão além de uma abordagem de avaliação centrada no indivíduo devem ser consideradas.

Essas atividades estão atualmente ausentes dos PTCs [27]. As contribuições para o desenvolvimento da carreira dos pesquisadores, em seus diferentes estágios são essenciais, bem como as contribuições de vários comitês relacionados à pesquisa (e.g., assumindo o papel de editor). A melhor forma de incorporá-las sem criar mais barreiras e burocracia, entretanto, tem sido debatida há bastante tempo [85].

Qualquer sistema de recompensas que tenha o empreendimento científico como elemento central e almeje promover um ambiente propício ao desenvolvimento de pesquisas confiáveis e úteis - com o mais alto grau de atenção à integridade em pesquisa - deve desenvolver estratégias para incorporar essas funções vitais em sua estrutura de avaliação. Esse fator ganha especial relevância se consideramos que ser um bom exemplo na ciência bem como orientar adequadamente pesquisadores em início de carreira são as principais prioridades na promoção da integridade em pesquisa [86].

\section{Implementações em curso}

A Universidade Macquarie, em Sydney, Austrália, tem algumas iniciativas interessantes em sua nova política de promoção acadêmica, que inclui cinco pilares, sendo um deles liderança \& cidadania. Nesse caso, os pesquisadores podem mostrar seu alinhamento com os valores da universidade e uma contribuição mais ampla para a instituição e sua comunidade [87]. Desde que essa política foi introduzida, foi relatado que o número de solicitações de promoção aumentou em $50 \%$, tendo também aumentado o número de mulheres promovidas [88].

Os critérios de promoção acadêmica da Universidade de Glasgow oferecem reconhecimento explícito para os pesquisadores que participam da revisão por pares [peer review] e outras atividades relacionadas (e.g., editoria de periódicos) [59,89]. Para tal, é necessário que haja organizações que forneçam aos revisores um identificador permanente (digital object identifier [DOI]), no caso de periódicos que publicam pareceres abertos [90]. Com esse DOI, as atividades de revisão podem ser incluídas nos currículos dos pesquisadores ou pareceres completos podem ser agrupados [91]. Essas políticas também podem fomentar um envolvimento mais significativo na capacitação para a revisão por pares [91]. A Universidade de Exeter, no Reino Unido, desenvolveu o "Exeter Academic", um núcleo de apoio para orientar seus pesquisadores quanto à progressão na carreira [92]. Liderança e cidadania são duas (de cinco) áreas principais. A primeira inclui mentoria e a última inclui caminhos para disseminar o conhecimento científico dos pesquisadores nas universidades.

O Conselho Consultivo Finlandês sobre Integridade em Pesquisa (Finnish Advisory Board on Research Integrity) (TENK) tem um modelo de currículos para pesquisadores com um amplo espectro de possibilidades contribuições, incluindo orientação e "confiança na sociedade" [93]. Como avaliação de orientação, a Universidade de Maastricht, na Holanda, considera a progressão na carreira de seus ex-doutorandos [94]. Não foi possível identificar instituições de pesquisa que reconhecem a contribuição de pesquisadores que participam de cursos de treinamento para a condução de uma orientação de alta qualidade [27]. 
A Agência Irlandesa de Pesquisa em Saúde (Irish Health Research Board) (HRB) tem um programa de financiamento para intercâmbio e disseminação de conhecimento. A HRB oferece aos pesquisadores financiados pela agência a oportunidade de buscar financiamentos adicionais para atividades de intercâmbio e disseminação de conhecimento que possam acelerar e maximizar o potencial translacional e o impacto dos resultados de pesquisa, bem como o aprendizado adquirido, sobre políticas ou práticas e sobre os resultados na saúde [95]. Há um sistema semelhante nos Institutos Canadenses de Pesquisa em Saúde [96] e no NC3Rs Skills and Knowledge Transfer grants [97], com a plataforma de inovação aberta Crack IT [98].

Os formulários de concessão de financiamento da Wellcome limitam o número de publicações que os candidatos podem enviar e propõem, de forma explícita, que seus candidatos detalhem suas outras produções. Além disso, há uma clara orientação para os avaliadores lembrando-os da importância de adotar uma visão ampla ao avaliar indivíduos [99].

\section{DISCUSSÃO}

O foco dos HKPs está na promoção de práticas de avaliação que fortaleçam a integridade em pesquisa, concentrando-se, deliberada e principalmente, no que as instituições de pesquisa podem fazer para modificar os critérios usados pelos PTCs para avaliações de carreira.

A ênfase em práticas de pesquisa responsáveis e na prevenção de práticas danosas à ciência é importante, uma vez que essas atitudes consomem tempo e recursos, podendo resultar em um número menor de projetos financiados e de publicações. Os HKPs sinalizam claramente que práticas fomentadoras da integridade em pesquisa precisam ser reconhecidas e recompensadas. Os cinco princípios que formulamos visam orientar o modo como as instituições de pesquisa devem incentivar, recompensar e avaliar os pesquisadores, individualmente, por atitudes que promovem a integridade em pesquisa em suas respectivas organizações.

Os HKPs não tratam de aspectos sobre gênero e outras formas de diversidade, inclusão ou questões associadas. Esses temas requerem uma avaliação por um grupo de pesquisadores (e.g., instituição de pesquisa) ao tomarem decisões sobre a alocação de financiamento ou políticas de recursos humanos. Além disso, esses tópicos dizem mais respeito à justiça social e à relevância social da pesquisa do que à integridade em pesquisa.

\section{Divulgação}

A Fundação para as Conferências Mundiais sobre Integridade em Pesquisa (WCRI Foundation) [100] e a Aliança REWARD (REduce research Waste And Review Diligence) [101] disponibilizarão os HKPs em seus websites. Esses sites incluirão os princípios, os signatários, os infográficos, as traduções em vários idiomas (em andamento), os planos de implementação futuros (em andamento) e, principalmente, um local para destacar aqueles que endossam os HKPs. Além da publicação em periódicos, estamos desenvolvendo outros meios sinérgicos de divulgação.

\section{Endosso e Adesão}

As instituições de pesquisa são fundamentais para os HKPs. Elas são o locus dos atuais e futuros pesquisadores, onde são realizadas avaliações de progressão na carreira e de produtividade. Para facilitar a implementação dos HKPs, na prática ["no chão das instituições"], o envolvimento das 
principais lideranças locais e o endosso do documento devem ser parte de seus planejamentos institucionais. Os HKPs foram reconhecidos pelo Conselho Diretor da Fundação WCRI e pelo Comitê Gestor da Aliança REWARD. Convidamos instituições acadêmicas, financiadores, outros grupos e indivíduos a fazerem o mesmo no website da Fundação WCRI, https://wcrif.org/guidance/hong-kongprinciples.

Convidamos também indivíduos e organizações para contribuírem com breves depoimentos (2-3 minutos) no YouTube sobre como vêm implementando os HKPs (categorizados pelo tipo de ator social/grupo de interesse) e para discutirem como integram os HKPs, em suas próprias iniciativas, e em outras. Incluiremos um link para esses vídeos no website da WCRI Foundation. Essa abordagem pode servir como uma medida pragmática para indivíduos e organizações indicarem de que forma estão endossando e aplicando os HKPs, inclusive para que possam estimular que outros tenham a mesma iniciativa.

A implementação de alguns desses princípios é provavelmente simples, embora não seja o caso para todos eles. Para tal, é necessário que haja uma maior compreensão sobre a complexidade dos ambientes de pesquisa atuais, como a disponibilidade de infraestrutura institucional. Também é necessário verificar se os formatos atuais de currículos são adequados para possibilitar a coleta de best practices, permitindo a transparência quanto à avaliação de carreira e considerando um maior alinhamento com as políticas dos financiadores.

Gostaríamos de avaliar esta abordagem e desenvolver ferramentas para os interessados em alternativas para implementar os cinco princípios. Trabalharemos com os signatários para levar essa iniciativa adiante. Entendemos que os HKPs são um grande passo nos esforços para fomentar a integridade em pesquisa e encorajamos um diálogo contínuo para apoiar a implementação desses importantes princípios.

\section{Agradecimentos}

Agradecemos aos muitos participantes da $6^{\text {a }}$ Conferência Mundial sobre Integridade em Pesquisa que forneceram feedback sobre as versões anteriores do documento e participaram ativamente das sessões de discussão durante a conferência. Agradecemos a Raymond Daniel pela ajuda na construção da base de dados da lista de referências.

\section{Citação sugerida para a tradução em língua portuguesa:}

[ABNT] MOHER, David et al. Princípios de Hong Kong para a Avaliação de Pesquisadores: Promovendo a Integridade em Pesquisa. Tradução: Sonia Vasconcelos, Carmen Penido, Sigmar de Mello Rode. ABEC Brasil, Botucatu, SP, v. 1, p. 01-20, jan./dez. 2021. Disponível em: https://doi.org/10.21452/abec.2021.abec.001. Título Original: The Hong Kong Principles for assessing researchers: Fostering research integrity. Disponível em: https://doi.org/10.1371/journal.pbio.3000737

[Vancouver] Moher D, Bouter L, Kleinert S, Glasziou P, Sham MH, Barbour V, et al. https://doi.org/10.1371/journal.pbio.3000737. Princípios de Hong Kong para a avaliação de pesquisadores: promovendo a integridade em pesquisa. Tradução de Sonia Vasconcelos, Carmen Penido, Sigmar de Mello Rode. ABEC Brasil 1: 01-20, 2021. https://doi.org/10.21452/abec.2021.abec.001. 


\section{REFERÊNCIAS}

1. Funk C, Hefferon M, Kennedy B, Johnson C. Pew Research Centre. Trust and Mistrust in Americans' Views of Scientific Experts [Internet]. Disponível em:

https://www.pewresearch.org/science/2019/08/02/trust-and-mistrust-in-americans-views-ofscientific-experts/. [25 mar. 2020]

2. Moher D, Naudet F, Cristea IA, Miedema F, Ioannidis JPA, Goodman SN. Assessing scientists for hiring, promotion, and tenure. PLoS Biol. 2018 Mar;16[3]:e2004089. pmid:29596415

Disponível no PubMed/NCBI Google Scholar

3. National Academies of Sciences Engineering and Medicine. Fostering Integrity in Research. Washington, DC: The National Academies Press; 2017.

4. American Society for Cell Biology. DORA. Declaration on Research Assessment [Internet]. Disponível em: http://www.ascb.org/dora/. [25 mar. 2020]

5. Hicks D, Wouters P, Waltman L, de RS, Rafols I. Bibliometrics: The Leiden Manifesto for research metrics. Nature. 2015 Apr 23;520[7548]:429-31. pmid:25903611

Disponível no PubMed/NCBI Google Scholar

6. Kretser A, Murphy D, Bertuzzi S, Abraham T, Allison DB, Boor KJ, et al. Scientific Integrity Principles and Best Practices: Recommendations from a Scientific Integrity Consortium. Sci Eng Ethics. 2019 Apr;25[2]:327-55. pmid:30810892

Disponível no PubMed/NCBI Google Scholar

7. Biagioli M, Lippman A. Gaming the Metrics. Misconduct and Manipulation in Academic Research. Cambridge, MA: MIT Press; 2020.

8. Leung LTF, Loock CA, Courtemanche R, Courtemanche DJ. A Cross-Sectional Analysis of the BC Children's Hospital Cleft Palate Program Waitlist. Plast Surg [Oakv]. 2019 Nov;27[4]:311-8.

Disponível no Google Scholar

9. Zauner H, Nogoy N, Edmunds S, Zhou H, Goodman L. Editorial: We need to talk about authorship. Gigascience. 2018;7[12]:1-4.

Disponível no Google Scholar

10. Quan W, Chen B, Shu F. Publish or impoverish: An investigation of the monetary reward system of science in China [1999-2016]. Aslib Journal of Information Management. 2017 Jan 1;69[5]:486-502.

Disponível no Google Scholar

11. Osterloh M, Frey BS. Ranking games. Eval Rev. 2015 Feb;39[1]:102-29. pmid:25092865 Disponível no PubMed/NCBI Google Scholar 
12. Canadian Institutes of Health Research. Strategy for Patient-Oriented Research [Internet]. Disponível em: https://cihr-irsc.gc.ca/e/41204.html. [25 mar. 2020]

13. Engaging with patients on research [Full Supplement]. CMAJ. 2018;190[Suppl.7]. Disponível no Google Scholar

14. The James Lind Alliance [Internet]. Disponível em: http://www.jla.nihr.ac.uk/. [25 mar. 2020]

15. Boote J, Dalgleish M, Freeman J, Jones Z, Miles M, Rodgers H. But is it a question worth asking? A reflective case study describing how public involvement can lead to researchers' ideas being abandoned. Health Expect. 2012;17[3]:440-51. pmid:22646745

Disponível no PubMed/NCBI Google Scholar

16. Shirk JL, Ballard HL, Wilderman CC, Phillips T, Wiggins A, Jordan R, et al. Public Participation in Scientific Research: a Framework for Deliberate Design. Ecology Society. $2012 ; 17[2]$.

Disponível no Google Scholar

17. Enhancing Reproducibility through Rigor and Transparency [Internet]. Disponível em: https://grants.nih.gov/policy/reproducibility/index.htm. [25 mar. 2020]

18. NC3Rs. The Experimental Design Assistant_EDA [Internet]. Disponível em: https://www.nc3rs.org.uk/experimental-design-assistant-eda. [3 abr. 2020]

19. Roberts Lab - School of Aquatic and Fishery Sciences. University of Washington [Internet]. Disponível em: http://faculty.washington.edu/sr320. [3 abr. 2020]

20. Cabrera, D. Mayo Clinic includes Social Media Scholarship Activities in Academic Advancement [Internet]. Disponível em: https://socialmedia.mayoclinic.org/2016/05/25/mayo-clinic-includes-social-mediascholarship-activities-in-academic-advancement/. [25 mar. 2020]

21. Wellcome's approach to engaging the public is going to change [Internet]. Disponível em: https://wellcome.ac.uk/news/wellcomes-approach-engaging-public-going-change. [25 mar. 2020]

22. Eyding D, Lelgemann M, Grouven U, Harter M, Kromp M, Kaiser T, et al. Reboxetine for acute treatment of major depression: systematic review and meta-analysis of published and unpublished placebo and selective serotonin reuptake inhibitor controlled trials. BMJ. 2010 Oct 12;341:c4737. pmid:20940209

Disponível no PubMed/NCBI Google Scholar

23. Franco A, Malhotra N, Simonovits G. Publication bias in the social sciences: Unlocking the file drawer. Science. 2014;345[6203]:1502-5. pmid:25170047

Disponível no PubMed/NCBI Google Scholar 
24. O'Boyle E, Banks G, Gonzalez-Mulé E. The Chrysalis Effect: How Ugly Initial Results Metamorphosize Into Beautiful Articles. Journal of Management 2020;43[2]:376-99. Disponível no Google Scholar

25. Chan AW, Song F, Vickers A, Jefferson T, Dickersin K, Gotzsche PC, et al. Increasing value and reducing waste: addressing inaccessible research. Lancet. 2014 Jan 18;383[9913]:257-66. pmid:24411650

Disponível no PubMed/NCBI Google Scholar

26. Dwan K, Gamble C, Williamson PR, Kirkham JJ. Systematic review of the empirical evidence of study publication bias and outcome reporting bias - an updated review. PLoS ONE. 2013;8[7]:e66844. pmid:23861749

Disponível no PubMed/NCBI Google Scholar

27. Rice, DB, Faffoul, H, Ioannidis, JPA, Moher, D. Academic criteria for promotion and tenure in faculties of biomedical sciences: a cross-sectional analysis of 146 universities [Internet]. Disponível em: https://doi.org/10.1101/802850. [25 mar. 2020]

28. Cobo E, Cortes J, Ribera JM, Cardellach F, Selva-O'Callaghan A, Kostov B, et al. Effect of using reporting guidelines during peer review on quality of final manuscripts submitted to a biomedical journal: masked randomised trial. BMJ. 2011 Nov 22;343:d6783. pmid:22108262

Disponível no PubMed/NCBI Google Scholar

29. Turner L, Shamseer L, Altman DG, Weeks L, Peters J, Kober T, et al. Consolidated standards of reporting trials [CONSORT] and the completeness of reporting of randomised controlled trials [RCTs] published in medical journals. Cochrane Database Syst Rev. 2012 Nov 14;11:MR000030. pmid:23152285

Disponível no PubMed/NCBI Google Scholar

30. Tunis AS, McInnes MD, Hanna R, Esmail K. Association of study quality with completeness of reporting: have completeness of reporting and quality of systematic reviews and meta-analyses in major radiology journals changed since publication of the PRISMA statement? Radiology. 2013 Nov;269[2]:413-26. pmid:23824992

Disponível no PubMed/NCBI Google Scholar

31. Korevaar DA, Wang J, van Enst WA, Leeflang MM, Hooft L, Smidt N, et al. Reporting diagnostic accuracy studies: some improvements after 10 years of STARD. Radiology. 2015 Mar;274[3]:781-9. pmid:25350641

Disponível no PubMed/NCBI Google Scholar

32. Wellcome Open Research. Policies [Internet]. Disponível em: https://wellcomeopenresearch.org/about/policies. [25 mar. 2020] 
33. Rapid \& Transparent Publishing [Internet]. Disponível em: https://gatesopenresearch.org/. [25 mar. 2020]

34. Maximising the 3Rs impact of NC3Rs-funded research [Internet]. Disponível em: https://f1000research.com/nc3rs. [25 mar. 2020]

35. Rapid \& Transparent Publishing [Internet]. Disponível em: https://amrcopenresearch.org. [25 mar. 2020]

36. Wellcome Open Research. Preparing a Registered Report [Internet]. Disponível em: https://wellcomeopenresearch.org/for-authors/article-guidelines/registered-reports. [3 abr. 2020]

37. Center for Open Science. What funders are doing to support transparent and reproducible research [Internet]. Disponível em: https://cos.io/top-funders/. [3 abr. 2020]

38. Center for Open Science. Registered Reports [Internet]. Disponível em: https://cos.io/rr/. [26 abr. 2020]

39. NIHR policy on clinical trial registration and disclosure of results [Internet]. Disponível em: https://www.nihr.ac.uk/about-us/documents/NIHR-Policy-on-Clinical-Trial-Registrationand-Disclosure-of-Results.pdf. National Institute for Health Research. [25 mar. 2020]

40. Strech D, Weissgerber T, Dirnagl U. Improving the trustworthiness, usefulness, and ethics of biomedical research through an innovative and comprehensive institutional initiative. PLoS Biol. 2020 Feb;18[2]:e3000576. pmid:32045410

Disponível no PubMed/NCBI Google Scholar

41. Open Science at Universities [Internet]. Disponível em: https://osf.io/kgnva/wiki/Universities/. [25 mar. 2020]

42. Welcome to the SPIRIT Statement website [Internet]. Disponível em: https://www.spiritstatement.org/. [25 mar. 2020]

43. Wicherts JM, Veldkamp CL, Augusteijn HE, Bakker M, van Aert RC, van Assen MA. Degrees of Freedom in Planning, Running, Analyzing, and Reporting Psychological Studies: A Checklist to Avoid p-Hacking. Front Psychol. 2016;7:1832. pmid:27933012 Disponível no PubMed/NCBI Google Scholar

44. Nosek BA, Ebersole CR, DeHaven AC, Mellor DT. The preregistration revolution. Proc Natl Acad Sci USA. 2018 Mar 13;115[11]:2600-6. pmid:29531091

Disponível no PubMed/NCBI Google Scholar

45. Allen C, Mehler DMA. Open science challenges, benefits and tips in early career and beyond. PLoS Biol. 2019 May;17[5]:e3000246. pmid:31042704

Disponível no PubMed/NCBI Google Scholar

46. Liberati A. An unfinished trip through uncertainties. BMJ. 2004;328[531]. 
Disponível no Google Scholar

47. Welcome to Open Lab Notebooks [Internet]. Disponível em: https://openlabnotebooks.org/. [25 mar. 2020]

48. Brand A, Allen L, Altman M, Hlava M, Scott J. Beyond authorship: attribution, contribution, collaboration, and credit. Learned Publishing. 2015;28[2]:151-5.

Disponível no Google Scholar

49. Naudet F, Sakarovitch C, Janiaud P, Cristea I, Fanelli D, Moher D, et al. Data sharing and reanalysis of randomized controlled trials in leading biomedical journals with a full data sharing policy: survey of studies published in The BMJ and PLOS Medicine. BMJ. 2018 Feb 13;360:k400. pmid:29440066

Disponível no PubMed/NCBI Google Scholar

50. Mello MM, Lieou V, Goodman SN. Clinical Trial Participants' Views of the Risks and Benefits of Data Sharing. N Engl J Med. 2018 Jun 7;378[23]:2202-11. pmid:29874542 Disponível no PubMed/NCBI Google Scholar

51. Munafo M, Nosek B, Bishop D, Button K, Chambers C, Percie du Sert N, et al. A manifesto for reproducible science. Nature Human Behaviour. 2017;1[1]:0021.

Disponível no Google Scholar

52. ASAPbio-Accelerating Science and Publication in biology [Internet]. Disponível em: https://asapbio.org/. [25 mar. 2020]

53. National Academies of Sciences Engineering and Medicine. Open Science by Design: Realizing a Vision for 21st Century Research. Washington, DC: The National Academies Press; 2018.

54. Using indicators in the evaluation of research [Internet]. Disponível em: https://www.ugent.be/en/research/research-ugent/research-strategy/indicators.htm. [25 mar. 2020]

55. Plan S. Making full and immediate Open Access a reality [Internet]. Disponível em: https://www.coalition-s.org/. [25 mar. 2020]

56. Research data [Internet]. Disponível em: https://www.data.cam.ac.uk/intro-data-champions. [25 mar. 2020]

57. Wilkinson MD, Dumontier M, Aalbersberg IJ, Appleton G, Axton M, Baak A, et al. The FAIR Guiding Principles for scientific data management and stewardship. Sci Data. 2016 Mar 15;3:160018. pmid:26978244

Disponível no PubMed/NCBI Google Scholar

58. Data Champions rewards [Internet]. Disponível em: 
https://www.tudelft.nl/en/library/current-topics/research-data-management/r/support/datachampions/our-data-champions/. [25 mar. 2020]

59. Academic Promotion Criteria Research Scientist Grades 7-9 [Internet]. Disponível em: https://www.gla.ac.uk/media/Media_498056_smxx.pdf. [25 mar. 2020]

60. Data Availability [Internet]. Disponível em: https://journals.plos.org/plosone/s/dataavailability. [25 mar. 2020]

61. TOP Standards [Internet]. Disponível em: https://www.topfactor.org/. [25 mar. 2020]

62. UK Orcid [Internet]. Disponível em: https://ukorcidsupport.jisc.ac.uk/2018/12/funders-signup-to-orcid-open-letter/. [25 mar. 2020]

63. Reporting Preprints and Other Interim Research Products [Internet]. Disponível em: https:/grants.nih.gov/grants/guide/notice-files/not-od-17-050.html. [25 mar. 2020]

64. Preprints [Internet]. Disponível em: https://mrc.ukri.org/research/policies-and-guidance-forresearchers/preprints/. [25 mar. 2020]

65. A more positive culture for $\mathrm{PhD}$ training [Internet]. Disponível em: https://wellcome.ac.uk/news/more-positive-culture-phd-training. [25 mar. 2020]

66. Amon A. A case for more curiosity-driven basic research. Mol Biol Cell. 2015 Nov 1;26[21]:3690-1. pmid:26515972

Disponível no PubMed/NCBI Google Scholar

67. Graphene [Internet]. Disponível em: https://www.graphene.manchester.ac.uk/learn/discovery-of-graphene/. [25 mar. 2020]

68. New Frontiers in Research Fund [Internet]. Disponível em: https://www.sshrccrsh.gc.ca/funding-financement/nfrf-fnfr/index-eng.aspx. [25 mar. 2020]

69. Camerer CF, Dreber A, Holzmeister F, Ho TH, Huber J, Johannesson M, et al. Evaluating the replicability of social science experiments in Nature and Science between 2010 and 2015. Nat Hum Behav. 2018 Sep;2[9]:637-44. pmid:31346273

Disponível no PubMed/NCBI Google Scholar

70. Why Meta-Research Matters [Internet]. Disponível em: https://metrics.stanford.edu/. [25 mar. 2020]

71. Quest [Internet]. Disponível em: https://www.bihealth.org/en/quest-center/missionapproaches/. [25 mar. 2020]

72. Meta Research Center [Internet]. Disponível em: https://metaresearch.nl. [25 mar. 2020]

73. Open Science Literature [Internet]. Disponível em: https://osf.io/kgnva/wiki/Open\%20Science\%20Literature/. [25 mar. 2020]

74. Intelligent technology to track research and evidence impact [Internet]. Disponível em: https://www.researchfish.net/. [25 mar. 2020] 
75. How we've defined what success looks like for Wellcome's work [Internet]. Disponível em: https://wellcome.ac.uk/news/how-weve-defined-what-success-looks-wellcomes-work. [25 mar. 2020]

76. Bert B, Heinl C, Chmielewska J, Schwarz F, Grune B, Hensel A, et al. Refining animal research: The Animal Study Registry. PLoS Biol. 2019 Oct;17[10]:e3000463. pmid:31613875

Disponível no PubMed/NCBI Google Scholar

77. New data re-use prizes help unlock the value of research [Internet]. Disponível em: https://wellcome.ac.uk/news/new-data-re-use-prizes-help-unlock-value-research. [25 mar. 2020]

78. Find grants awarded [Internet]. Disponível em: https://wellcome.ac.uk/funding/people-andprojects/grants-awarded?scheme_id = 3569. [25 mar. 2020]

79. Replication Studies [Internet]. Disponível em: https://bit.ly/2H1PIt3. [25 mar. 2020]

80. Meta-Research: Evaluation and Scientometrics [Internet] Disponível em: https://collections.plos.org/meta-research-evaluation-and-scientometrics. [25 mar. 2020]

81. Meta-Research: A Collection of Articles [Internet]. Disponível em: https://elifesciences.org/collections/8d233d47/meta-research-a-collection-of-articles. [25 mar. 2020]

82. NIHR policy on clinical trial registration and disclosure of results [Internet]. Disponível em: https://www.nihr.ac.uk/about-us/documents/NIHR-Policy-on-Clinical-Trial-Registrationand-Disclosure-of-Results.pdf. [25 mar. 2020]

83. Funding scheme priority areas [Internet]. Disponível em: https://www.nc3rs.org.uk/fundingscheme-priority-areas. [25 mar. 2020]

84. The CAMARADES/ NC3Rs Systematic Review Facility [SyRF] [Internet]. Disponível em: https://www.nc3rs.org.uk/camaradesnc3rs-systematic-review-facility-syrf. [25 mar. 2020]

85. Credit for Peer Review: What is it Worth? [Internet]. Disponível em: https://scholarlykitchen.sspnet.org/2018/10/18/credit-for-peer-review-what-exactly-doesthat-mean/. [25 mar. 2020]

86. Haven T, Tijdink J, Pasman H, Widdershoven G, ter Riet G, Bouter L. Do research misbehaviours differ between disciplinary fields? A mixed methods study among academic researchers in Amsterdam. Res Integrity Peer Rev. 2019;4[25].

Disponível no Google Scholar

87. Macquarie University. New Academic Promotion scheme [Internet]. Disponível em: https://www.mq.edu.au/thisweek/2017/04/13/new-academic-promotionscheme/\#.XnvhhYhKg2x. [25 mar. 2020] 
88. Macquarie University. Towards inclusive academic promotion. https://figshare.com/articles/EPHEA_Wollongong_Hughes_pptx/12331517. [2020 May 21]

89. Boyer, EL. Scholarship reconsidered: Priorities of the professoriate [Internet]. Disponível em: https://www.umces.edu/sites/default/files/al/pdfs/BoyerScholarshipReconsidered.pdf. [25 mar. 2020]

90. Reviewer Guidelines [Internet]. Disponível em: https://f1000research.com/forreferees/guidelines. [25 mar. 2020]

91. Track more of your research impact [Internet]. Disponível em: https://publons.com/about/home. [25 mar. 2020]

92. Exeter Academic. Your development [Internet]. Disponível em: http://www.exeter.ac.uk/staff/exeteracademic/yourdevelopment/. [25 mar. 2020]

93. A template for a researcher's curriculum vitae [Internet]. Disponível em: https://www.tenk.fi/sites/tenk.fi/files/CV_english_270613.pdf. [26 mar. 2020]

94. de Goede, M, Belder, R, and de Jonge, J. Academic careers in the Netherlands [Internet]. Disponível em: https://www.rathenau.nl/sites/default/files/201805/Facts_and_Figures_Academic_Careers_01.pdf. [26 mar. 2020]

95. Platforms, programmes and projects [Internet]. Disponível em: https://www.hrb.ie/funding/funding-awarded/platforms-programmes-and-projects/. [26 mar. 2020]

96. Population and public health. IPPH funding [Internet]. Disponível em: https://cihrirsc.gc.ca/e/46949.html. [26 mar. 2020]

97. Skills and Knowledge Transfer grants [Internet]. Disponível em: https://www.nc3rs.org.uk/skills-and-knowledge-transfer-grants. [26 mar. 2020]

98. The CRACK IT innovation platform [Internet]. Disponível em: https://nc3rs.org.uk/crackit/. [26 mar. 2020]

99. Induction pack for committee members [Internet]. Disponível em: https://wellcome.ac.uk/sites/default/files/induction-pack-for-committee-members-2018.pdf. [26 mar. 2020]

100. The World Conferences on Research Integrity [Internet]. Disponível em: https://www.wcrif.org/. [26 mar. 2020]

101. The Reward Alliance [Internet] Disponível em: http://rewardalliance.net/. [26 mar. 2020] 